\title{
Global plankton ecosystem functions emerging from fundamental constraints of individual cells
}

\author{
KEN H. ANDERSEN
}

Technical University of Denmark

Presenting Author:kha@aqua.dtu.dk

Unicellular plankton span all three domains of life: primary producers, herbi-/carnivores, and decomposers (bacteria). However, individual cells often fill more than one of these functional roles, e.g., by combining phototrophy with osmoheterotrophy or phagotrophy. This diversity in function at the cell level complicates ecosystem models. The complexity can be reduced by models organized around cell size. Here I review the size-based approach to plankton modelling. I focus on the parameterization of the fundamental mechanisms at the level of the individual cell and how they can be related to fundamental constraints from geometry, fluid mechanics and chemical reaction kinetics. The emergent functions at the cell level scales directly up to the function of the entire unicellular plankton ecosystem: primary production, respiration and losses, and carbon available to production of higher trophic levels. Sizebased simulation models rely on a small set of generic parameters that are universal across regions and changing climate. They are therefore particularly suitable for global-scale simulations and I show results of global scale assessment of ecosystem functions related to biogeochemical cycles. Finally, I present a generic library to easily apply and embed size-based models in model systems: chemostats, water columns, and global circulation models. 